This document is the Accepted Manuscript version of a Published Work that appeared in final form in ACS Applied Interfaces, (c) 2016 American Chemical Society after peer review and technical editing by publisher. To access the final edited and published work see Zhang, Z., \& Liu, J. (2016). Molecularly Imprinted Polymers with DNA Aptamer Fragments as Macromonomers. Acs Applied Materials \& Interfaces, 8(10), 6371-6378. https://doi.org/10.1021/acsami.6b00461

\title{
Molecular Imprinted Polymers with DNA Aptamer Fragments as Macromonomers
}

Zijie Zhang and Juewen Liu*

Department of Chemistry, Waterloo Institute for Nanotechnology

University of Waterloo

Waterloo, Ontario, N2L 3G1, Canada

Phone: (+1) 5198884567 ext. 38919

Email: liujw@uwaterloo.ca 


\begin{abstract}
Molecularly imprinted polymers (MIPs) are produced in the presence of a template molecule. After removing the template, the cavity can selectively rebind the template. MIPs are attractive functional materials with a low cost and high stability, but traditional MIPs often suffer from low binding affinity. This study employs DNA aptamer fragments as macromonomers to improve MIPs. The DNA aptamer for adenosine was first split into two halves, fluorescently labeled, and co-polymerized into MIPs. With a fluorescence quenching assay, the importance of imprinting was confirmed. Further studies were carried out using isothermal titration calorimetry (ITC). Compared to the mixture of the free aptamer fragments, their MIPs doubled the binding affinity. Each free aptamer fragment alone cannot bind adenosine, while MIPs containing each fragment are effective binders. We further shortened one of the aptamer fragments, and the DNA length was pushed to as short as six nucleotides, yielding MIPs with a dissociation constant of $27 \mu \mathrm{M}$ adenosine. This study provides a new method for preparing functional MIP materials by combining high affinity biopolymer fragments with low cost synthetic monomers, allowing higher binding affinity and providing a method for signaling binding based on DNA chemistry.
\end{abstract}

Keywords: aptamers; molecular imprinting; polymers; adenosine; fluorescence; isothermal titration calorimetry 


\section{Introduction}

Molecular recognition is critically important in biology, biomaterials science, analytical chemistry, and medicine. A few types of biologically derived molecules, such as antibodies and aptamers, are general platforms for molecular recognition. ${ }^{1-4}$ While these biopolymers possess high binding affinity and specificity, they are expensive, often unstable, and prone to denaturation. Molecularly imprinted polymers (MIPs) are prepared by polymerizing small molecule synthetic monomers around target molecules to form binding cavities, such that the cavities can rebind the target. ${ }^{5}$ Compared to antibodies and aptamers, MIPs can be prepared in much larger quantities at a lower cost with higher stability. ${ }^{6,7}$ Many different targets have been imprinted including small molecules, peptides, and proteins. ${ }^{8-10}$ As a result, MIPs are attractive for a wide range of applications in analytical chemistry, separation, and environmental remediation. ${ }^{11,12}$ MIPs have been made with various types of monomers, mostly of which are acrylic and silane based. ${ }^{13,14}$ However, most MIPs suffer from poor binding affinity or specificity. ${ }^{15,16}$ At the same time, it is difficult to produce a fluorescence signal for binding.

We are interested in exploring the feasibility of combining biopolymers and MIPs by introducing a fragment of biological ligands as macromonomers in MIPs. DNA aptamers are oligonucleotides that selectively bind to target molecules. ${ }^{17,18}$ DNA aptamers are obtained from combinatorial selections and they can be evolved to bind many target molecules ${ }^{19,20}$ Incorporation of full-length aptamers in MIPs was previously reported for thrombin binding, ${ }^{21}$ and recently for prostate specific antigen via terminal acrydite modification of the aptamers. ${ }^{22}$ Acrylamidemodified uridines were incorporated into the cocaine aptamer, so that polymerization took place at multiple positions along the aptamer chain. ${ }^{23}$ 
In all the previous cases, full-length aptamers were used. Our idea is to use aptamer fragments as macromonomers to improve MIPs, and then gradually shorten the aptamer fragment length. Shorter fragments allow a lower cost and better stability. We hypothesize that good binding might still be achieved with the help of the MIP matrix. For easy handling and good template accessibility, MIP hydrogel nanoparticles (or nanogels) were prepared in this study. ${ }^{24-31}$

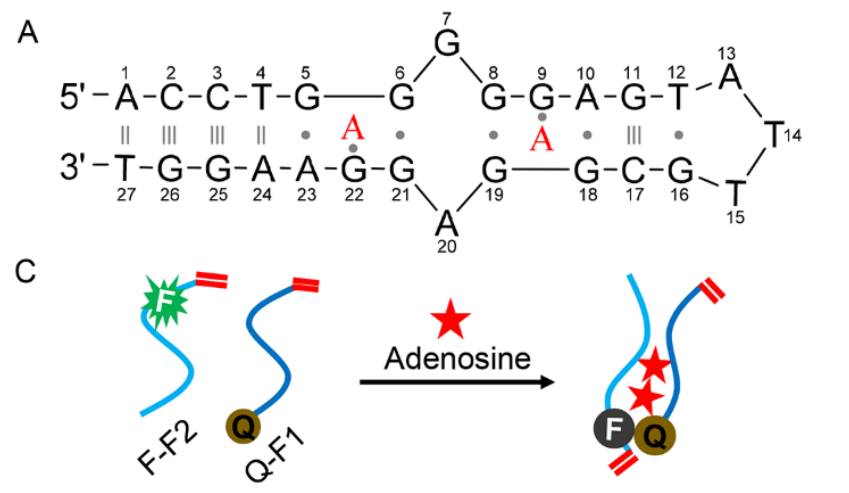

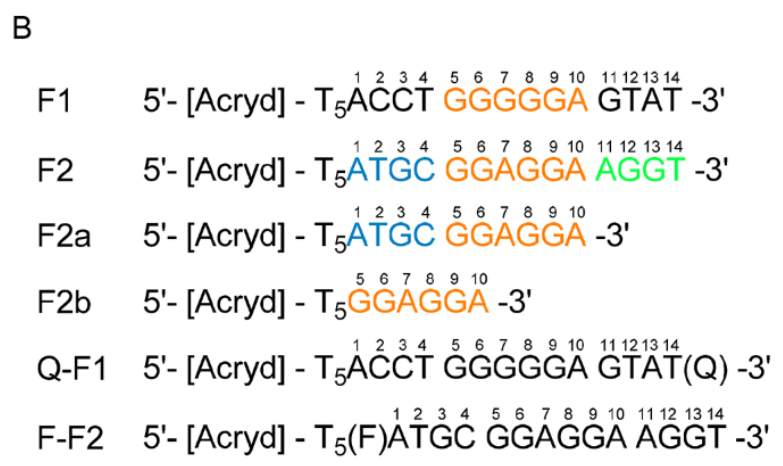

D
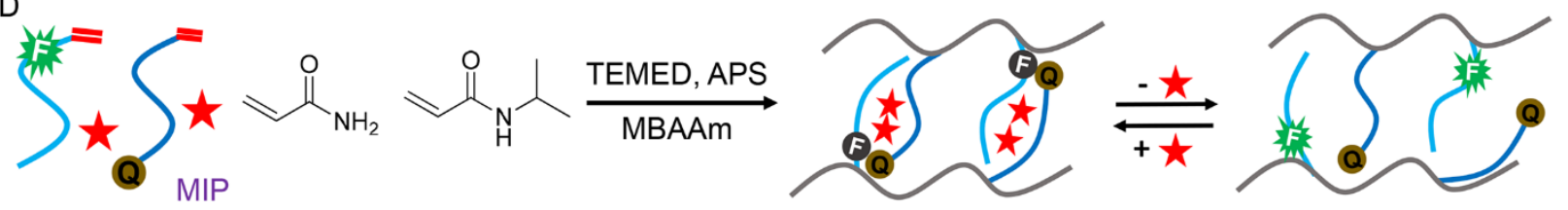

$\mathrm{E}$
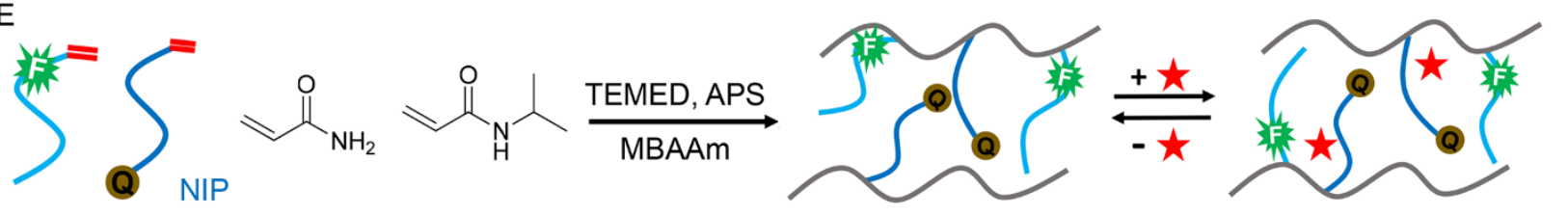

Figure 1. (A) The secondary structure of the full-length adenosine aptamer binding two adenosine molecules (the 'A' in red). (B) The sequences of the adenosine aptamer fragments used in this work named F1, F2, F2a, F2b, Q-F1 (Black Hole Quencher 1 (Q) labeled), and F-F2 (carboxyfluorescein, FAM (F) labeled), respectively, all modified with a 5'-acrydite. (C) Schematic presentation of the two adenosine aptamer fragments (F-F2 and Q-F1) binding adenosine and fluorescence quenching. Schematic presentation of (D) adenosine imprinted (MIP) 
and (E) non-imprinted (NIP) MIPs with the aptamer fragments. TEMED and APS are used to initiate the polymerization reaction. MBAAm is the crosslinker.

\section{Materials and Methods}

Chemicals. All of the DNA samples were purchased from Eurofins (Huntsville, AL). Acrylamide (AAm), N-isopropylacrylamide (NIPAAm), methylene bisacrylamide (MBAAm), and sodium dodecyl sulfate (SDS) were from Sigma-Aldrich. Ammonium persulphate (APS) and N,N,N',N'tetramethylethylenediamine (TEMED) were from VWR. Sodium chloride, magnesium chloride, 4-(2-hydroxyethyl)-1-piperazineethanesulfonic acid (HEPES) and adenosine and cytidine were from Mandel Scientific (Guelph, Ontario, Canada). Mill-Q water was used to prepare all the buffers and solutions. All other reagents and solvents were of analytical grade and used as received.

Preparation of nanogels. All the nanogels were prepared using the aqueous precipitation polymerization method with same concentrations of monomers, crosslinker, and initiator to achieve maximal consistency. Optimization of gel formulation was reported previously, ${ }^{32}$ and is not repeated here. For the nanogels containing fluorophore (or quencher)-labeled aptamers, AAm ( $2.9 \mathrm{mg}, 42 \mu \mathrm{mol})$, NIPAAm ( $4.6 \mathrm{mg}, 42 \mu \mathrm{mol})$, aptamer fragments ( $20 \mu \mathrm{M}$ each) and the template molecule adenosine or cytidine ( $1 \mathrm{mM})$ were dissolved in buffer A (50 mM HEPES, pH 7.6, 100 $\mathrm{mM} \mathrm{NaCl}, 5 \mathrm{mM} \mathrm{MgCl}$ ). The mixture was incubated for $30 \mathrm{~min}$ with slow stirring at $25^{\circ} \mathrm{C}$ to form binding complexes. Then the crosslinker MBAAm $(2.4 \mathrm{mg}, 16 \mu \mathrm{mol})$ and surfactant SDS $(0.8 \mathrm{mg})$ were added. After purging the mixture with $\mathrm{N}_{2}$ for $1 \mathrm{~h}$, polymerization was initiated by adding APS $(0.6 \mathrm{mg})$ and TEMED $(0.3 \mu \mathrm{L})$. The final reaction volume was standardized to $1 \mathrm{~mL}$. The reaction was continued for $4 \mathrm{~h}$ at $25{ }^{\circ} \mathrm{C}$ under a $\mathrm{N}_{2}$ atmosphere. The resultant imprinted nanogels were collected by centrifugation at $15,000 \mathrm{rpm}$ for $10 \mathrm{~min}$ and then washed extensively 
using Milli-Q water until complete removal of the unreacted monomers and templates (confirmed by UV-Vis spectrometry). The gels were frozen-dried for $24 \mathrm{~h}$, and weighed to determine the reaction yield. The nanogels prepared for the ITC tests were prepared using the same method except that $70 \mu \mathrm{M}$ of aptamer fragments were used. A higher DNA concentration was used for ITC since this technique is less sensitive compared to fluorescence. Non-imprinted nanogels (NIPs) were also prepared and washed in the same way except that no template was added during polymerization. The concentration of the template adenosine was chosen to be $1 \mathrm{mM}$ to avoid stacking of adenosine forming multimers at high concentrations (adenosine has relatively low solubility).

Dynamic light scattering (DLS). The hydrodynamic sizes of the nanogels were measured by DLS (Zetasizer Nano ZS90, Malvern). Each nanogel sample $(50 \mu \mathrm{g} / \mathrm{mL})$ was dispersed in buffer A and the temperature was maintained at $25^{\circ} \mathrm{C}$ during measurement.

Coupling efficiency. The coupling efficiencies of the fluorophore-labeled aptamer incorporated nanogels were determined by fluorescence spectroscopy at $520 \mathrm{~nm}$. For other nanogels the coupling efficiencies were determined by UV-Vis absorbance at $260 \mathrm{~nm}$. After preparation, the nanogel samples were purified by centrifugation $(1,500 \mathrm{rpm}, 10 \mathrm{~min})$. The supernatant and a standard solution (containing the same initial aptamer concentration during preparation) were diluted to the same volume in buffer A. Their fluorescence emission or absorbance was measured to calculate coupling efficiency. Any DNA without an acrydite group will be washed away. ${ }^{33,34}$

Isothermal titration calorimetry (ITC). ITC was performed using a VP-ITC Microcalorimeter instrument (MicroCal). Prior to each measurement, each solution and suspension was degassed to avoid air bubbles. The aptamer fragments $(20 \mu \mathrm{M})$ or nanogel samples in buffer A was loaded in a $1.45 \mathrm{~mL}$ ITC cell at $25^{\circ} \mathrm{C}$. Adenosine $(280 \mu \mathrm{L}, 3 \mathrm{mM})$ in the same buffer was titrated into the 
cell through a syringe (10 $\mu \mathrm{L}$ each time, except for the first injection being $2 \mu \mathrm{L})$. The enthalpy $(\Delta H)$ and binding constant $\left(K_{\mathrm{a}}\right)$ were obtained through fitting the titration curves to a one-site binding model using the Origin software. The $K_{\mathrm{d}}$ values were calculated from $1 / K_{a}$ and $\Delta G=-$ $R T \ln \left(K_{a}\right)$, where $R$ is the gas constant. $\Delta S$ was calculated from $\Delta G=\Delta H-T \Delta S$.

\section{Results and Discussion}

\section{Molecular imprinting helps aptamer binding.}

The adenosine DNA aptamer has been extensively studied as a model for biosensor development. ${ }^{35-38}$ The full-length aptamer contains only 27 nucleotides (Figure 1A). It can be split into two halves and binding still occurs when both fragments are present (Figure 1B). ${ }^{39-43}$ We first tested the effect of molecular imprinting if both halves were used as macromonomers. Each aptamer fragment was labeled on the 5'-end with an acrydite group to co-polymerize into the gel.

To measure binding, one fragment was labeled with a dark quencher on its 3 '-end (Q-F1), and the other with an internal FAM (F-F2, Figure 1B; DNA sequences and modifications in Figure S1). Upon forming the aptamer complex, the fluorescence is expected to quench (Figure 1C). Two small molecule monomers, acrylamide and N-isopropylacrylamide (NIPAAm), were included to form the gel matrix, and bis-acrylamide (MBAAm) was used as a crosslinker.

To compare the imprinting effect, three types of nanogels were prepared by precipitation polymerization: ${ }^{32}$ one in the absence of any target (named NIP), one with $1 \mathrm{mM}$ cytidine (C-MIP), and the last one with $1 \mathrm{mM}$ adenosine (A-MIP). We reason that the aptamer binding complex might form in the presence of adenosine, positioning the two aptamer halves forming a high affinity cavity (Figure 1D). Without adenosine, polymerization is random and the resulting gel 
particles may not bind adenosine (Figure 1E). A similar outcome is expected when cytidine is added into the pre-polymerization mixture as it does not form complex with the aptamer monomers. All the gel particles have a similar size of $\sim 170 \mathrm{~nm}$ as determined by DLS and they are easily dispersed in water (Figure S2). Among the various nucleotides, we only chose cytidine here as a negative control since this adenosine aptamer is a well-studied model system. It has a similarly high affinity for adenosine, AMP, and ATP, but has no binding when the base part is changed to C, T, or G. ${ }^{35}$

The DNA coupling efficiency was determined to be $\sim 30 \%$ (Table S1) for all the gels (Figure S3). After washing away the template molecules and free monomers, the three gels were re-dispersed in buffer A with the same final aptamer concentration (128 nM, each fragment). Upon adding $1 \mathrm{mM}$ adenosine, the fluorescence intensity at $520 \mathrm{~nm}$ of the A-MIP gel decreased significantly (Figure 2C), suggesting the two aptamer halves reassembled. In comparison, the signal from the NIP (Figure 2A) and C-MIP (Figure 2B) gels only dropped slightly indicating the lack of aptamer binding. As a control, none of these samples were quenched by $1 \mathrm{mM}$ cytidine. This study indicates that imprinting during gel formation is critical for re-binding. We also measured the kinetics of binding and a stable signal was achieved in 1 min (Figure S4). Since the DNAs were immobilized, these gels can be re-used after washing away the adenosine (Figure S5).

To quantitatively understand binding, relative fluorescence quenching $\left(F / F_{0}\right)$ of these gels were measured as a function of adenosine concentration (Figure 2D). With increasing adenosine concentration, the signal from the NIP and C-MIP decreased slightly reaching a final ratio around 0.80, while a final ratio of 0.46 was achieved for the A-MIP. We also compared the same concentration of the free split aptamers without any gel (Figure 2D, blue trace). Interestingly, it only decayed to a final ratio of 0.66 . The dissociation constant $\left(K_{\mathrm{d}}\right)$ of the free aptamer fragments 
$(1.6 \mathrm{mM})$ is four times higher than that for the A-MIP gels $(0.4 \mathrm{mM})$. Therefore, with imprinting, the split aptamer fragments are positioned at a more favorable binding configuration than the free aptamers in solution. ${ }^{44}$ This improved binding is also related to an increased effective concentration of the aptamers by linking them to the gel matrix. In this regard, the gel matrix helped aptamer binding.

From this initial test, we confirmed that imprinting indeed took place during preparation of these gels. Without adenosine, the two aptamer fragments were randomly distributed during polymerization. Confined by the crosslinked gel network, they may not reach each other in the presence of adenosine (Figure 1E). The presence of fluorescence signal in this method allows for convenient detection of adenosine, which is a significant advantage brought by DNA.
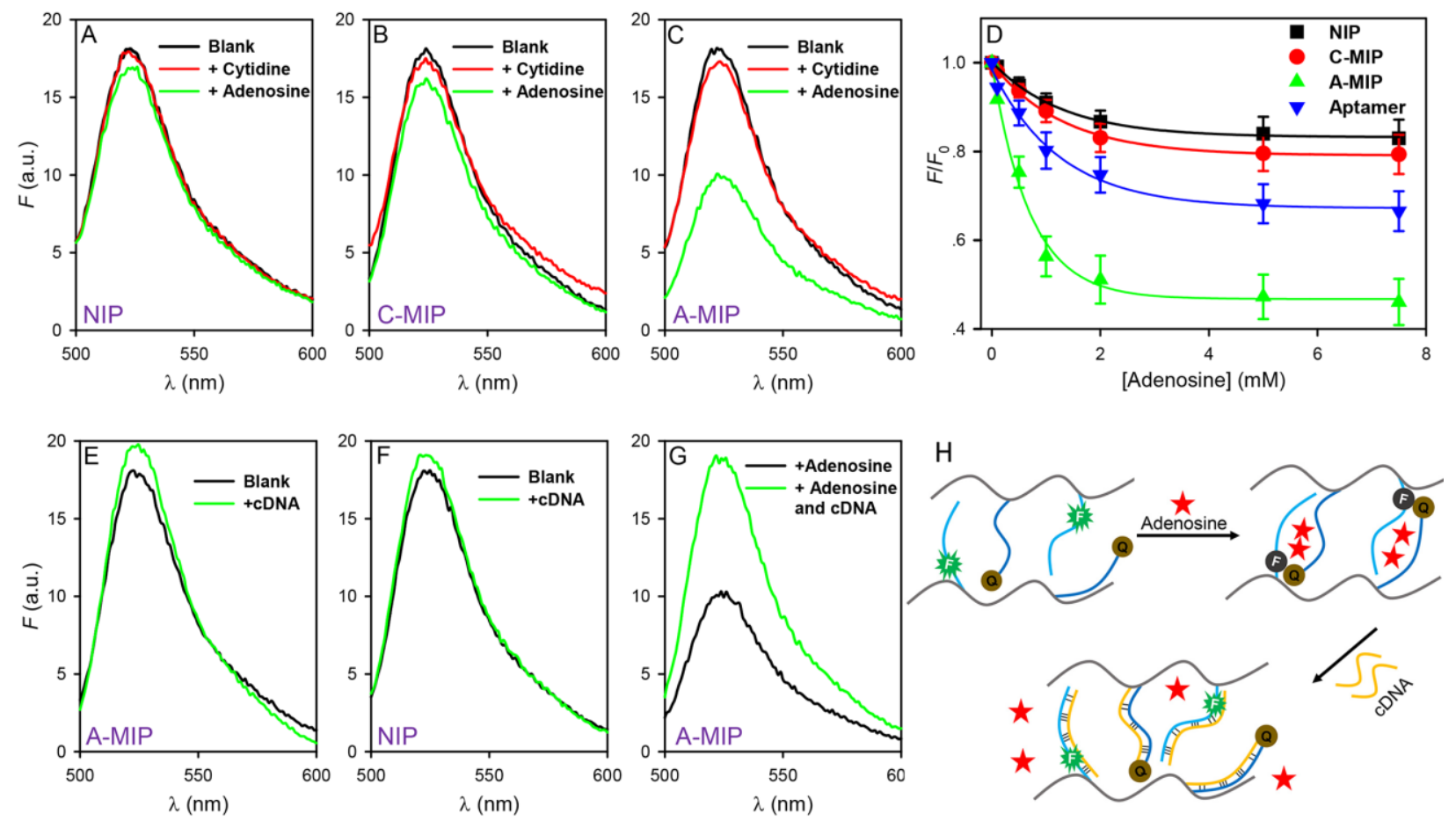

Figure 2. Fluorescence spectra for (A) the NIP, (B) the C-MIP and (C) the A-MIP gels before and after adding $1 \mathrm{mM}$ cytidine or adenosine. (D) Fluorescence quenching of the three gels and the free split aptamer (128 nM, each fragment) at $520 \mathrm{~nm}$ as a function of adenosine concentration. 
Fluorescence spectra for (E) the A-MIP and (F) the NIP gels after adding the two cDNAs (128 nM each) of the two aptamer fragments. (G) Fluorescence spectra of the A-MIP gels with $1 \mathrm{mM}$ adenosine and then adding the cDNAs. $(\mathrm{H})$ Schematic presentation of using the cDNAs to open the aptamer binding complex. For all the experiments, the gels $(0.13 \mathrm{mg} / \mathrm{mL})$ all contained a final of $128 \mathrm{nM}$ aptamers in buffer A (50 mM HEPES, pH 7.6, $100 \mathrm{mM} \mathrm{NaCl}, 5 \mathrm{mM} \mathrm{MgCl}$ ).

\section{Probing the initial aptamer binding complex.}

Since the two aptamer halves can partially base pair with each other (Figure 1A, B), it is possible that a fraction of the aptamers are already hybridized in the nanogels in the absence of adenosine. For this population, further addition of adenosine may not induce further fluorescence quenching. Therefore, it is important to measure the fraction of the initial binding complex in the gels. For this purpose, we designed an experiment as shown in Figure 2H. The complementary DNAs (cDNAs) of the two aptamer fragments were added to the gels to disrupt the aptamer binding structures and to increase fluorescence. For the A-MIP sample without adenosine, the cDNAs led to a slight fluorescence increase of $\sim 10 \%$ (Figure 2E); for the NIP gel, the increase was only $\sim 5 \%$ (Figure 2F). The free DNA (no gel) after binding with its cDNA also has a slight fluorescence of 3.3\% (Figure S6). Based on this, we conclude that $2 \%$ initial binding complexes formed for the NIP, while $\sim 7 \%$ of the aptamers were in the complex form for the A-MIP in the absence of adenosine. This is reasonable since the aptamer fragments in the A-MIP were closer to each other due to imprinting. As a positive control, we add adenosine to the A-MIP gel first. We then observed a large fluorescence increase of $\sim 80 \%$ after adding the cDNAs (Figure $2 \mathrm{G}$ ). Therefore, most of aptamer fragments did not form the binding complex in the absence of adenosine. This is important for the analytical applications to achieve a large signal change. 


\section{Free individual aptamer fragments cannot bind.}

With both aptamer halves, it is not surprising that the A-MIP gel binds adenosine. A more challenging question is to take a piece of DNA that cannot bind adenosine for imprinting. To test this, we started with each aptamer fragment for imprinting. Since this design makes fluorescencebased assay difficult, we employed non-labeled DNA and characterized binding using isothermal titration calorimetry (ITC). The two fragments are named F1 and F2, respectively (Figure 1B).

When adenosine was titrated into either aptamer fragment (F1 or F2, no gels), barely any heat was detected (Figure $3 \mathrm{~A}$ and $\mathrm{B}$ ), suggesting no binding by the individual fragments. On the other hand, a strong binding was detected for the sample containing both fragments (F1 plus F2, no gels, Figure 3C). The thermodynamic values of the above experiments were calculated (Table 1). The enthalpy change $(\Delta H)$ for each aptamer fragment binding adenosine was at least 18 -fold lower than that when both fragments were used. Since the heat was very small, we cannot obtain an accurate fitting for other thermodynamic values. Based on the NMR structure of the aptamer, ${ }^{35,36}$ both halves contribute indispensable purine bases (G9 and G22) and base stacking to stabilize the G.A pair for binding the target adenosine (Figure 1A). It is not surprising that each fragment alone failed to bind. 


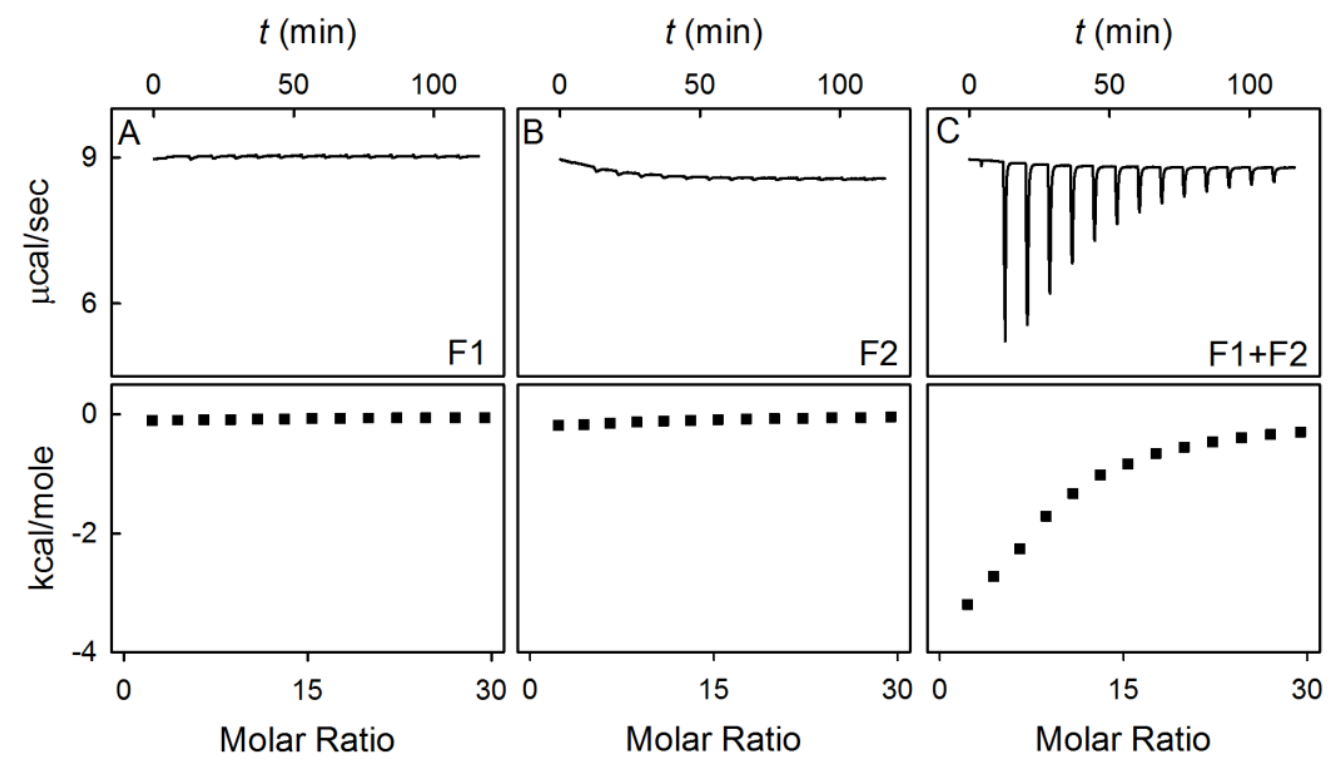

Figure 3. ITC titration curves obtained at $298 \mathrm{~K}$ for binding between $20 \mu \mathrm{M}$ of (A) F1, (B) F2 and (C) F1+F2 with $3 \mathrm{mM}$ adenosine in buffer A. The original titration traces (top) and the integrated heat (bottom) of each reaction are shown.

Table 1. Thermodynamic data for adenosine titrating to the aptamer fragments by ITC.

\begin{tabular}{llllll}
\hline Aptamers & $\boldsymbol{K}_{\mathbf{a}}$ & $\boldsymbol{K}_{\mathbf{d}}$ & $\boldsymbol{\Delta} \boldsymbol{G}$ & $\boldsymbol{\Delta H}$ & $\boldsymbol{\Delta S}$ \\
& $\left(\times 10^{4} \mathrm{M}^{-1}\right)$ & $(\mu \mathrm{M})$ & $\left(\mathrm{kcal} \mathrm{mol}^{-1}\right)$ & $\begin{array}{l}\left(\mathrm{kcal} \mathrm{mol}^{-1}\right) \\
\left(\mathrm{cal} \mathrm{K}^{-1} \mathrm{~mol}^{-1}\right)\end{array}$ \\
\hline F1 & - & - & - & $-0.11 \pm 0.04$ & - \\
F2 & - & - & - & $-0.18 \pm 0.07$ & - \\
F1+F2 & $3.05 \pm 0.22$ & $32.7 \pm 2.4$ & -6.1 & $-3.19 \pm 0.6$ & 9.9
\end{tabular}

Imprinting with individual aptamer fragments.

After confirming the lack of binding for the individual free aptamer fragments, we next tested whether imprinting can rescue their binding. We hypothesize that some monomers in the gel may serve as the other half of the aptamer to form an effective binding complex. Here a few new gels 
were prepared: no DNA imprinted with adenosine (MIP), or non-imprinted (NIP); with either aptamer fragment imprinted (F1-MIP and F2-MIP), or non-imprinted (F1-NIP and F2-NIP); and with both aptamer fragments imprinted (F1F2-MIP), or non-imprinted (F1F2-NIP). Each aptamer fragment had a 5'-acrydite for co-polymerization into the gel matrix (coupling efficiencies in Table S1 and Figure S7).

In contrast to the free individual aptamer fragments that cannot bind adenosine (Figure 3A and B, Table 1), the imprinted gels with either fragment (F1-MIP and F2-MIP) released significant heat indicating rescued binding activity (Figure 4B and C, Table 2). Compared to the imprinted gel without aptamer (Figure 4A), the F1-MIP gel has increased binding affinity by 6.5-fold, and the F2-MIP gel by 13.8-fold. Therefore, using DNA oligomers that alone cannot bind adenosine, the imprinted polymer has drastically improved affinity. Note that the DNA oligomers added during polymerization was only $70 \mu \mathrm{M}$, which was 1400 -fold lower in concentration than the synthetic monomers. The fact that the DNA can still effect binding indicates the tightest binding sites are associated with DNA.

Between the two aptamer fragments, the F2 containing gels (F2-MIP) had a $K_{\text {a value }} \sim 2$ fold larger than that of the F1-MIP. Therefore, DNA sequence still play an important role. This F1 fragment contains 5 consecutive guanines, which may promote intramolecular interactions and thus weakening adenosine binding during imprinting. DNA binding adenosine can take place via hydrogen bonding, base stacking, and hydrophobic interactions. At this moment, it is unclear whether the binding is the same as that in the original aptamer for each half aptamer, or via other interactions. Based on this experiment, for a given target without known aptamers, it is likely that a careful DNA sequence design is needed; not every sequence is equal. For comparison, we also made the same gels but without adenosine imprinting (Figure 4E-H, Table 2). In each case, the 
amount of heat was significantly less compared to their imprinted counterpart, indicating the importance of imprinting and specific binding in the imprinted gels.

It needs to be noted that we plotted Figure $4 \mathrm{~A}$ and $4 \mathrm{E}$ in the same way as the rest, even though these two samples did not contain any DNA. Therefore, the molar ratio in their $x$-axis is not an accurate representation of the number of binding cavities in the gels. It is likely that these

It is also interesting to note that the $K_{\mathrm{d}}$ measured here $(16 \mu \mathrm{M}$ adenosine for F1F2-MIP) using ITC is much tighter than that from fluorescence $(0.4 \mathrm{mM})$. This is attributable to the higher DNA concentration used for preparing gels for ITC $(70 \mu \mathrm{M})$ than for fluorescence $(20 \mu \mathrm{M})$. Since both fragments are involved in binding, the final binding complex is tripartite (containing both fragments and adenosine). As a result, the DNA concentration difference is reflected in the apparent $K_{\mathrm{d}}$ measured (e.g. the 3.5-fold difference in DNA concentration leads to $3.5 \times 3.5=12.25$ fold difference in $K_{\mathrm{d}}$ ). Considering the difference in coupling efficiency, the DNA final concentration induced effect should be $\sim 16$-fold, which is close to our observation of 25 -fold. For comparison, the full aptamer (without splitting) has a $K_{\mathrm{d}}$ of $\sim 6 \mu \mathrm{M} .^{35}$

From this study, we could confirm that imprinting rescued the binding activity of the aptamer fragments. While the binding of each individual fragment alone is not as strong as that with both fragments used together, it supports the feasibility of using DNA oligomers as macromonomers in MIPs. 


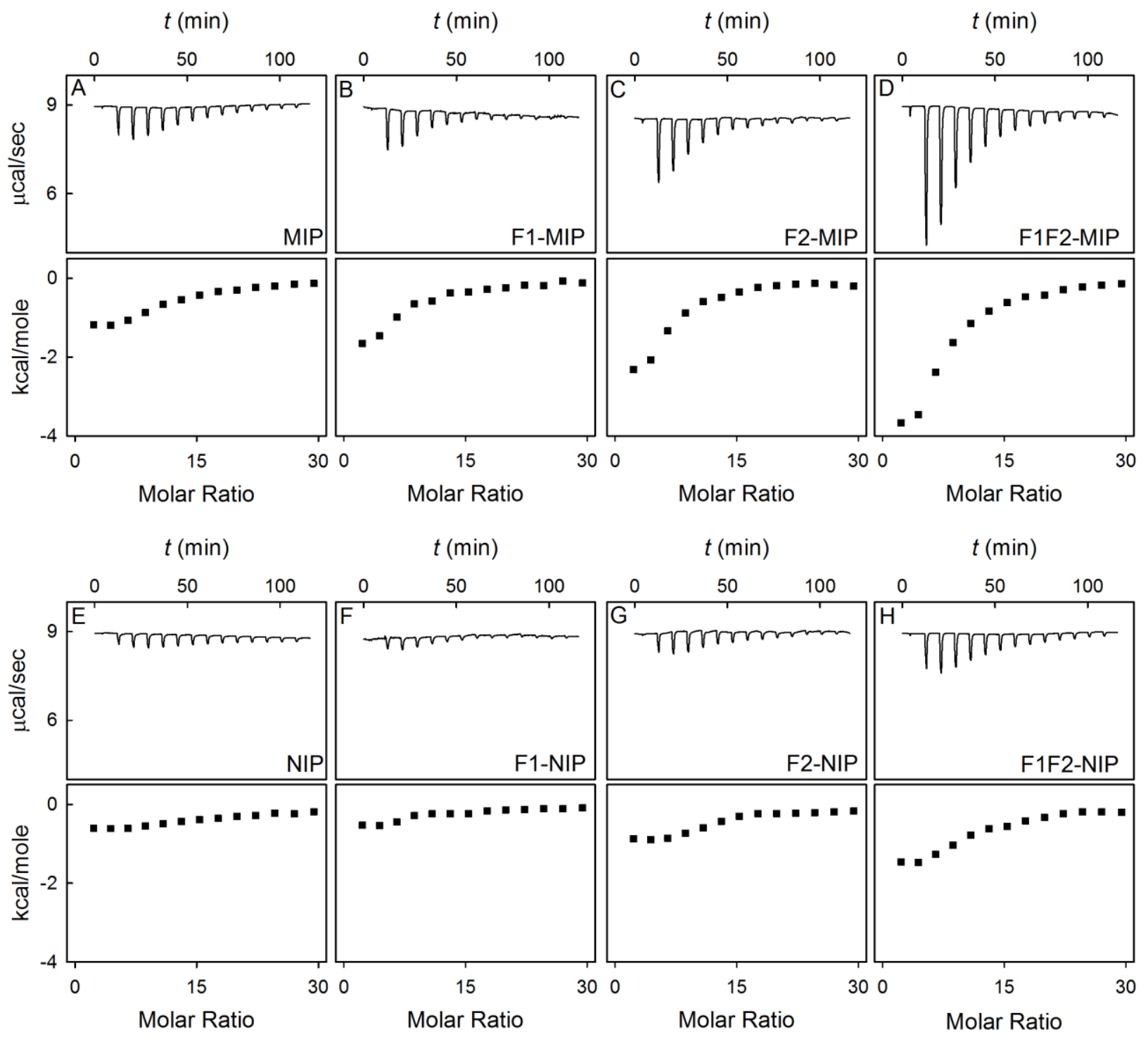

Figure 4. ITC titration curves obtained at $298 \mathrm{~K}$ for binding of adenosine by the (A) MIP, (B) F1MIP, (C) F2-MIP, (D) F1F2-MIP, (E) NIP, (F) F1-NIP, (G) F2-NIP and (H) F1F2-NIP. The gel concentrations are around $6 \mathrm{mg} / \mathrm{mL}$; individually adjusted to ensure that each sample contains 20 $\mu \mathrm{M}$ DNA (if imprinted with DNA). The adenosine stock concentration was $3 \mathrm{mM}$. The original titration traces (top) and the integrated heat (below) of each reaction are shown. 
Table 2. Binding data for adenosine titrating to nanogels by ITC.

\begin{tabular}{|c|c|c|c|c|c|}
\hline Nanogels & $\begin{array}{l}\boldsymbol{K}_{\mathbf{a}} \\
\left(\times 10^{4} \mathrm{M}^{-1}\right)\end{array}$ & $\begin{array}{l}\boldsymbol{K}_{\mathbf{d}} \\
(\mu \mathrm{M})\end{array}$ & $\begin{array}{l}\Delta G \\
\left(\mathrm{kcal} \mathrm{mol}^{-1}\right)\end{array}$ & $\begin{array}{l}\boldsymbol{\Delta H} \\
\left(\mathrm{kcal} \mathrm{mol}^{-1}\right)\end{array}$ & $\begin{array}{l}\Delta S \\
\left(\mathrm{cal} \mathrm{K}^{-1} \mathrm{~mol}^{-1}\right)\end{array}$ \\
\hline MIP & $0.4 \pm 0.05$ & $250 \pm 31$ & -4.8 & $-1.18 \pm 0.4$ & 12.2 \\
\hline F1-MIP & $2.6 \pm 0.2$ & $38 \pm 3.0$ & -6.0 & $-1.65 \pm 0.2$ & 14.7 \\
\hline F2-MIP & $5.5 \pm 0.4$ & $18 \pm 1.3$ & -6.4 & $-2.31 \pm 0.2$ & 13.9 \\
\hline F1F2-MIP & $6.3 \pm 0.6$ & $16 \pm 1.5$ & -6.5 & $-3.67 \pm 0.6$ & 9.64 \\
\hline NIP & $0.06 \pm 0.01$ & $1670 \pm 197$ & -3.7 & $-0.60 \pm 0.1$ & 10.4 \\
\hline F1-NIP & $0.3 \pm 0.06$ & $330 \pm 69$ & -4.8 & $-0.53 \pm 0.06$ & 14.4 \\
\hline F2-NIP & $0.8 \pm 0.1$ & $125 \pm 16$ & -5.3 & $-0.88 \pm 0.05$ & 14.8 \\
\hline F1F2-NIP & $1.1 \pm 0.1$ & $91 \pm 8$ & -5.5 & $-1.46 \pm 0.4$ & 13.6 \\
\hline
\end{tabular}

\section{Imprinting using shorter aptamer fragments.}

After knowing that each aptamer fragment alone can be effective in the MIPs, it is interesting to test even shorter DNA sequences. According to the structure of the aptamer, the middle six nucleotides in F2 (from G5 to A10) are primarily responsible for specific adenosine binding, while its two flanking segments contribute only to the overall aptamer folding (Figure 1A and B). Hence we further shorten the F2 fragment to make F2a and F2b (Figure 1B). To test the binding of each DNA, a few new gels incorporating these sequences were prepared (Figure S1), and their binding to adenosine was characterized by ITC. For the F2a imprinted gels (F2a-MIP), a considerable amount of heat was detected with a high binding constant (Figure 5A and Table 3). These values are comparable with the full fragment 2 gel (F2-MIP, Figure 5C), indicating that the truncated nucleotides from A11 to T14 may not contribute much to adenosine binding even in the MIP gels. To push the limit, we further shorten the F2a to design F2b with only six nucleotides (Figure 1B). 
For the imprinted gels containing F2b (F2b-MIP), their binding affinities decreased compared with full F2 fragment but still keep a high value of $K_{\mathrm{a}}$ (Figure 5B and C, Table 3). We reason that the six nucleotides from G5 to A10 are the main contributors for binding adenosine. Since neighboring bases can influence the stacking energy, ${ }^{45,46}$ the moderate loss in binding may be from the loss of base stacking on this end. As controls, their corresponding NIP gels barely showed any heat release (Figure 5D-F, and Table 3).

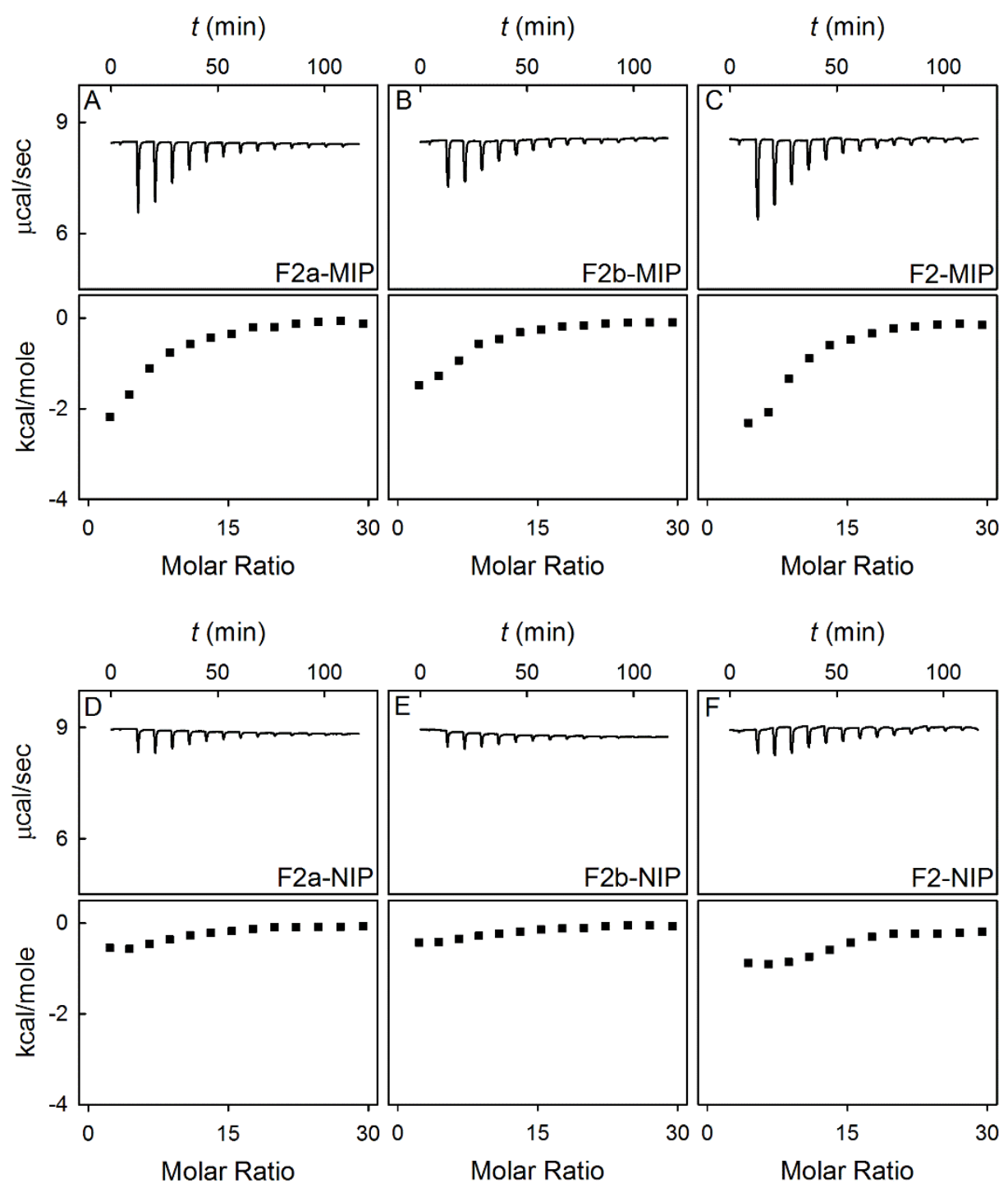


Figure 5. ITC titration curves obtained at $298 \mathrm{~K}$ for binding between adenosine $(3 \mathrm{mM})$ with the (A) F2a-MIP, (B) F2b-MIP, (C) F2-MIP, (D) F2a-NIP, (E) F2b-NIP, and (F) F2-NIP. For all the experiments, the nanogels all contained $20 \mu \mathrm{M}$ aptamers (gel $=\sim 6.0 \mathrm{mg} / \mathrm{mL}$ ) in buffer A. The original titration traces (top) and the integrated heat (below) of each reaction are shown respectively.

In this work, adenosine was chosen as a model target and it happens to be a nucleoside, which may have more interactions with DNA (e.g. via base stacking and base pairing). Given the development of the aptamer field, we believe this method can also be applied to other target molecules as well. For example, the cocaine aptamer has a similar binding affinity, ${ }^{47,48}$ while cocaine does not resemble a nucleotide.

The full-length aptamer (27-nucleotide) was finally shortened to six nucleotides, while tight binding was still retained. This represents a substantial save in the cost of DNA synthesis (scales linearly with DNA length) with higher yield and purity (scales with a power law with DNA length). Most importantly, it indicates that a low concentration of DNA can have a large influence on the binding property of MIPs.

Table 3. Binding data for adenosine titrating to nanogels by ITC.

\begin{tabular}{llllll}
\hline Nanogels & $\boldsymbol{K}_{\mathbf{a}}$ & $\begin{array}{l}\boldsymbol{K}_{\mathbf{d}} \\
\left(\times 10^{4} \mathrm{M}^{-1}\right)\end{array}$ & $\begin{array}{l}\Delta \boldsymbol{G} \\
(\mu \mathrm{M})\end{array}$ & $\begin{array}{l}\boldsymbol{\Delta} \boldsymbol{k} \\
\left(\mathrm{kcal} \mathrm{mol} \mathrm{mol}^{-1}\right)\end{array}$ & $\begin{array}{l}\Delta \boldsymbol{S} \\
\left(\mathrm{cal} \mathrm{K}^{-1} \mathrm{~mol}^{-1}\right)\end{array}$ \\
\hline F2a-MIP & $5.1 \pm 0.4$ & $19 \pm 1.5$ & -6.4 & $-2.18 \pm 0.6$ & 14.1 \\
F2b- MIP & $3.6 \pm 0.2$ & $27 \pm 1.6$ & -6.2 & $-1.48 \pm 0.4$ & 15.8 \\
F2-MIP & $5.5 \pm 0.4$ & $18 \pm 1.3$ & -6.4 & $-2.31 \pm 0.2$ & 13.9 \\
F2a-NIP & $0.4 \pm 0.06$ & $250 \pm 38$ & -4.8 & $-0.55 \pm 0.06$ & 14.3
\end{tabular}




$\begin{array}{llllll}\text { F2b-NIP } & 0.6 \pm 0.05 & 170 \pm 14 & -5.1 & -0.43 \pm 0.08 & 15.7 \\ \text { F2-NIP } & 0.8 \pm 0.1 & 125 \pm 16 & -5.3 & -0.88 \pm 0.05 & 14.8\end{array}$

\section{Conclusions}

For the first time, we prepared new hybrid materials using aptamer fragments as macromonomers in MIPs. All the previous work used full-length aptamers to prepare MIPs. Using aptamer fragments can reduce the cost of DNA synthesis, while still improving the MIP binding and signaling properties. We showed that even for DNA sequences that cannot bind the target molecule, they can still be quite effective upon imprinting. Different DNA sequences perform differently in MIPs, and rational DNA sequence design is likely to be important for target molecules without known aptamers. We summarize our findings in the following aspects. 1) DNA help MIPs. Compared to the two synthetic monomers (acrylamide and $\mathrm{N}$-isopropylacrylamide), DNA macromonomers increased the binding affinity by up to 18 -fold. For optimal performance, the DNA sequence needs to be carefully designed and screened. 2) Imprinting helps aptamers. When both fragments are used, a fully functional aptamer can form. Compared to the free aptamer binding, the imprinted material has doubled the binding affinity by positioning the two fragments close to each other in an optimal configuration. This is consistent with previous reports using fulllength aptamers. 3) It might be possible to de novo design DNA sequences to optimally bind a given target molecule without known aptamers. In this aspect, computer modeling might be a powerful tool to predict DNA oligomer sequences. 4) DNA may also allow convenient signaling. Over the past two decades, the analytical chemistry of aptamer has significantly advanced. By introducing such knowledge to MIPs, new and better biosensors might be produced. Overall, combining DNA oligomers and MIPs is a promising method for obtaining new functional materials. 


\section{Supporting Information}

The Supporting Information is available free of charge on the ACS Publications website at http://pub.acs.org.

DLS, DNA coupling efficiency, binding kinetics, reversibility, and fluorescence enhancement control experiments.

\section{Acknowledgement}

Funding for this work is from the Natural Sciences and Engineering Research Council of Canada (NSERC), grant number: STPGP 447472-13.

\section{References}

(1) Hermann, T.; Patel, D. J. Adaptive Recognition by Nucleic Acid Aptamers. Science 2000, $287,820-825$.

(2) Liu, J.; Cao, Z.; Lu, Y. Functional Nucleic Acid Sensors. Chem. Rev. 2009, 109, 19481998.

(3) Wang, H.; Yang, R.; Yang, L.; Tan, W. Nucleic Acid Conjugated Nanomaterials for Enhanced Molecular Recognition. ACS Nano 2009, 3, 2451-2460.

(4) Sellergren, B. Noncovalent Molecular Imprinting: Antibody-like Molecular Recognition in Polymeric Network Materials. TrAC, Trends Anal. Chem. 1997, 16, 310-320. 
(5) Wulff, G.; Sarhan, A.; Zabrocki, K. Enzyme-Analogue Built Polymers and Their Use for the Resolution of Racemates. Tetrahedron Lett. 1973, 44, 4329-4332.

(6) Haupt, K.; Mosbach, K. Molecularly Imprinted Polymers and Their Use in Biomimetic Sensors. Chem. Rev. 2000, 100, 2495-2504.

(7) Ramström, O.; Ye, L.; Mosbach, K. Artificial Antibodies to Corticosteroids Prepared by Molecular Imprinting. Chem. Biol. 1996, 3, 471-477.

(8) Bossi, a; Bonini, F.; Turner, a P. F.; Piletsky, S. a. Molecularly Imprinted Polymers for the Recognition of Proteins: The State of the Art. Biosens. Bioelectron. 2007, 22, 11311137.

(9) Zhang, W.; Yang, F. K.; Han, Y.; Gaikwad, R.; Leonenko, Z.; Zhao, B. PDA35-Surface and Tribological Behaviors of the Bioinspired Polydopamine Thin Films under Dry and Wet Conditions. Biomacromolecules 2013, 14, 394-405.

(10) Matsui, J.; Akamatsu, K.; Hara, N.; Miyoshi, D.; Nawafune, H.; Tamaki, K.; Sugimoto, N. SPR Sensor Chip for Detection of Small Molecules Using Molecularly Imprinted Polymer with Embedded Gold Nanoparticles. Anal. Chem. 2005, 77, 4282-4285.

(11) Schirhagl, R. Bioapplications for Molecularly Imprinted Polymers. Anal. Chem. 2014, 86, $250-261$.

(12) Volkert, A. A.; Haes, A. J. Advancements in Nanosensors Using Plastic Antibodies. Analyst 2014, 139, 21-31.

(13) Whitcombe, M. J.; Chianella, I.; Larcombe, L.; Piletsky, S. a; Noble, J.; Porter, R.; Horgan, A. The Rational Development of Molecularly Imprinted Polymer-Based Sensors 
for Protein Detection. Chem. Soc. Rev. 2011, 40, 1547-1571.

(14) Shiomi, T.; Matsui, M.; Mizukami, F.; Sakaguchi, K. A Method for the Molecular Imprinting of Hemoglobin on Silica Surfaces Using Silanes. Biomaterials 2005, 26, 55645571.

(15) Alenazi, N. A.; Lai, E. P. C.; Manthorpe, J. M. Enhanced Selectivity of a Molecularly Imprinted Polymer toward the Target Molecule via Esterification of Non-Specific Binding Sites with Diazomethane. J. Mol. Recognit. 2014, 27, 755-762.

EL-Sharif, H. F.; Hawkins, D. M.; Stevenson, D.; Reddy, S. M. Determination of Protein Binding Affinities within Hydrogel-Based Molecularly Imprinted Polymers (HydroMIPs). Phys. Chem. Chem. Phys. 2014, 16, 15483-15489.

(17) Wilson, D. S.; Szostak, J. W. In Vitro Selection of Functional Nucleic Acids. Annu. Rev. Biochem. 1999, 51, 611-647.

(18) Famulok, M.; Hartig, J. S.; Mayer, G. Functional Aptamers and Aptazymes in Biotechnology, Diagnostics, and Therapy. Chem. Rev. 2007, 107, 3715-3743.

(19) Ellington, a D.; Szostak, J. W. In Vitro Selection of RNA Molecules That Bind Specific Ligands. Nature 1990, 346, 818-822.

(20) Tuerk, C.; Gold, L. Systematic Evolution of Ligands by Exponential Enrichment: RNA Ligands to Bacteriophage T4 DNA Polymerase. Science 1990, 249, 505-510.

(21) Bai, W.; Gariano, N. a.; Spivak, D. a. Macromolecular Amplification of Binding Response in Superaptamer Hydrogels. J. Am. Chem. Soc. 2013, 135, 6977-6984.

(22) Jolly, P.; Tamboli, V.; Harniman, R. L.; Estrela, P.; Allender, C. J.; Bowen, J. L. 
Aptamer-MIP Hybrid Receptor for Highly Sensitive Electrochemical Detection of Prostate Specific Antigen. Biosens. Bioelectron. 2016, 75, 188-195.

(23) Poma, A.; Brahmbhatt, H.; Pendergraff, H. M.; Watts, J. K.; Turner, N. W. Generation of Novel Hybrid Aptamer-Molecularly Imprinted Polymeric Nanoparticles. Adv. Mater. $\mathbf{2 0 1 5}, 27,750-758$.

(24) Peppas, N. A.; Hilt, J. Z.; Khademhosseini, A.; Langer, R. Hydrogels in Biology and Medicine: From Molecular Principles to Bionanotechnology. Adv. Mater. 2006, 18, 13451360.

(25) Li, S.; Gaddes, E. R.; Chen, N.; Wang, Y. Molecular Encryption and Reconfiguration for Remodeling of Dynamic Hydrogels. Angew. Chem., Int. Ed. 2015, 127, 6055-6059.

(26) Battig, M. R.; Soontornworajit, B.; Wang, Y. Programmable Release of Multiple Protein Drugs from Aptamer-Functionalized Hydrogels via Nucleic Acid Hybridization. J. Am. Chem. Soc. 2012, 134, 12410-12413.

(27) Yang, H.; Liu, H.; Kang, H.; Tan, W. Engineering Target-Responsive Hydrogels Based on Aptamer-Target Interactions. J. Am. Chem. Soc. 2008, 130, 6320-6321.

(28) Zhu, Z.; Wu, C.; Liu, H.; Zou, Y.; Zhang, X.; Kang, H.; Yang, C. J.; Tan, W. An Aptamer Cross-Linked Hydrogel as a Colorimetric Platform for Visual Detection. Angew. Chem., Int. Ed. 2010, 49, 1052-1056.

(29) Xing, Y.; Cheng, E.; Yang, Y.; Chen, P.; Zhang, T.; Sun, Y.; Yang, Z.; Liu, D. SelfAssembled DNA Hydrogels with Designable Thermal and Enzymatic Responsiveness. Adv. Mater. 2011, 23, 1117-1121. 
(30) Liu, J. Oligonucleotide-Functionalized Hydrogels as Stimuli Responsive Materials and Biosensors. Soft Matter 2011, 7, 6757-6767.

(31) Dave, N.; Chan, M. Y.; Huang, P. J. J.; Smith, B. D.; Liu, J. Regenerable DNAFunctionalized Hydrogels for Ultrasensitive, Instrument-Free mercury(II) Detection and Removal in Water. J. Am. Chem. Soc. 2010, 132, 12668-12673.

(32) Zhang, Z.; Liu, J. Improving Molecularly Imprinted Nanogels by pH Modulation. RSC Adv. 2015, 5, 91018-91025.

(33) Baeissa, A.; Dave, N.; Smith, B. D.; Liu, J. DNA-Functionalized Monolithic Hydrogels and Gold Nanoparticles for Colorimetric DNA Detection. ACS Appl. Mater. Interfaces 2010, 2, 3594-3600.

(34) Rehman, F. N.; Audeh, M.; Abrams, E. S.; Hammond, P. W.; Kenney, M.; Boles, T. C. Immobilization of Acrylamide-Modified Oligonucleotides by Co-Polymerization. Nucleic Acids Res. 1999, 27, 649-655.

(35) Huizenga, D. E.; Szostak, J. W. A DNA Aptamer That Binds Adenosine and ATP. Biochemistry 1995, 34, 656-665.

(36) Lin, C. H.; Patel, D. J. Structural Basis of DNA Folding and Recognition in an AMPDNA Aptamer Complex: Distinct Architectures but Common Recognition Motifs for DNA and RNA Aptamers Complexed to AMP. Chem. Biol. 1997, 4, 817-832.

(37) Liu, J.; Lu, Y. Fast Colorimetric Sensing of Adenosine and Cocaine Based on a General Sensor Design Involving Aptamers and Nanoparticles. Angew. Chem., Int. Ed. 2005, 45, 90-94. 
(38) Liu, H.; Xiang, Y.; Lu, Y.; Crooks, R. M. Aptamer-Based Origami Paper Analytical Device for Electrochemical Detection of Adenosine. Angew. Chem., Int. Ed. Engl. 2012, $51,6925-6928$.

(39) Stojanovic, M. N.; De Prada, P.; Landry, D. W. Fluorescent Sensors Based on Aptamer Self-Assembly. J. Am. Chem. Soc. 2000, 122, 11547-11548.

(40) Li, F.; Zhang, J.; Cao, X.; Wang, L.; Li, D.; Song, S.; Ye, B.; Fan, C. Adenosine Detection by Using Gold Nanoparticles and Designed Aptamer Sequences. Analyst 2009, 134, $1355-1360$.

(41) Sharma, A. K.; Heemstra, J. M. Small-Molecule-Dependent Split Aptamer Ligation. J. Am. Chem. Soc. 2011, 133, 12426-12429.

(42) Nguyen, T. H.; Steinbock, L. J.; Butt, H. J.; Helm, M.; Berger, R. Measuring Single Small Molecule Binding via Rupture Forces of a Split Aptamer. J. Am. Chem. Soc. 2011, 133, 2025-2027.

(43) Dave, N.; Liu, J. Biomimetic Sensing Based on Chemically Induced Assembly of a Signaling DNA Aptamer on a Fluid Bilayer Membrane. Chem. Commun. 2012, 48, 37183720.

(44) Liao, Y. J.; Shiang, Y. C.; Huang, C. C.; Chang, H. T. Molecularly Imprinted Aptamers of Gold Nanoparticles for the Enzymatic Inhibition and Detection of Thrombin. Langmuir 2012, 28, 8944-8951.

(45) Yakovchuk, P. Base-Stacking and Base-Pairing Contributions into Thermal Stability of the DNA Double Helix. Nucleic Acids Res. 2006, 34, 564-574. 
(46) Kool, E. T. Hydrogen Bonding, Base Stacking, and Steric Effects in DNA Replication. Annu. Rev. Biophys. Biomol. Struct. 2001, 30, 1-22.

(47) Stojanovic, M. N.; De Prada, P.; Landry, D. W. Aptamer-Based Folding Fluorescent Sensor for Cocaine. J. Am. Chem. Soc. 2001, 123, 4928-4931.

(48) Neves, M. A. D.; Reinstein, O.; Saad, M.; Johnson, P. E. Defining the Secondary Structural Requirements of a Cocaine-Binding Aptamer by a Thermodynamic and Mutation Study. Biophys. Chem. 2010, 153, 9-16. 